\title{
DERMATOFIBROSARCOMA PROTUBERANS ON THE BREAST: A CASE REPORTED AND DISCUSSION ABOUT THE STEPS FOR YOUR DIAGNOSIS
}

Fernanda Mabel Batista Aquino', Larissa Santos Lourenço Ferreira'1, Jader Rodrigues Gonçalves', Clarissa Amaral Abreu², Maria Isabel Oliveira Silva²

1 Liga Norte Riograndense Contra O Cancer - Natal (RN), Brazil.

${ }^{2}$ Universidade Potiguar - Natal (RN), Brazil.

Introduction: Dermatofibrosarcoma Protuberans (DFSP) is a soft tissue tumor, with slow growth and low metastasis rate, but with a high chance of local recurrence. Epidemiologically, DFSP represents only $0.1 \%$ of all malignant tumors. It is more common in women aged between 30 and 50 years and the most affected site is the trunk. However, the presentation in the breast is rare. Clinically, DFSP is revealed as a slow and asymptomatic growth nodule that originates in the dermis and invades the subcutaneous tissue. The diagnosis is made by histopathology, with the result of uniform spindle cells with low atypia or mitotic activity, and immunohistochemistry, demonstrating, mainly, positivity for the CD34 antibody, and negativity for the S100 antibody. The standard treatment of DFSP is a complete surgical resection with negative margins. However, the minimum resection margin to achieve local control remains undefined. The present study aims to discuss a case of DFSP with a rare presentation in the mammary region and the steps for its diagnosis. Case report: A 37-year-old woman with an exophytic and ulcerated lesion of $5 \mathrm{~cm}$ in the lower inner quadrant (LIQ) of the right breast (RB). The patient reported the appearance of a skin lesion 10 years before in the same location, evolving with a superficial nodule over the last five years and with an accelerated growth of the lesion one month before. There are no other tangible nodules in the breasts and no armpit lymph nodes. Breast ultrasound: exophytic and vascularized nodule in RB LIQ, of $8.4 \mathrm{~cm}$ - BIRADS 4. Histopathological report after lesion excision: spindle cell neoplasm of mesenchymal origin, without necrosis, measuring $5.8 \times 5.8 \mathrm{~cm}$, mitotic index: 03 mitoses in 10 high-rise fields. The immunohistochemistry of the excised lesion indicated positivity for the CD34 and Ki-67 antibodies and negativity for the S-100, CD99, Desmina and Myogenin antibodies. The immunohistochemical profile, associated with the morphological findings, corroborates the diagnosis of Dermatofibrosarcoma protuberans. Surgical margins indicated involvement by the neoplasm. Thereafter, a surgical approach was discussed and indicated, which was not accepted by the patient. 